\title{
Genetic Association with Coronary Artery Disease
}

\author{
Md. Toufiqur Rahman ${ }^{1}$, AKM Monwarul Islam² \\ ${ }^{1}$ Department of Cardiology, National Institute of Cardiovascular Diseases, Dhaka, ${ }^{2}$ Department of \\ Cardiology, Sir Salimullah Medical College.
}

Keywords: Genetics,

(Cardiovasc. j. 2017; 9(2): 159-166) coronary artery disease, Ischaemic heart disease.

\section{Introduction:}

Coronary artery disease (CAD) is still the numberone killer in the world, and clinical trials indicate that it is preventable. Mortality and morbidity can be reduced by at least $30 \%$ to $40 \%$ by treating known risk factors. Genetic susceptibility is claimed to account for $50 \%$ of predisposition. The challenge of preventing $\mathrm{CAD}$ in this century, as claimed by some investigators, will require a more comprehensive prevention and treatment of environmental and genetic risk factors. Part of that challenge has been met by genome-wide association studies, which have identified 36 genetic variants with increased risk for CAD. All of these genetic variants have reached genome-wide significance $(5 \times 10 " 8)$ and replicate in independent populations with large sample sizes. More than $50 \%$ of these variants occur in $>50 \%$ of the population, with 10 occurring in $>75 \%$ of the population. The challenge and the opportunity lie in the observation that $>66 \%$ of these risk variants do not mediate their risk through known conventional risk factors. These results suggest that genetic predisposition for CAD is conferred by common DNA variants and many factors contributing to the pathogenesis of $\mathrm{CAD}$ are yet to be determined. Comprehensive prevention of $\mathrm{CAD}$ will most likely require combating genetic and environmental risk factors. ${ }^{1,2}$

During the last decade there was remarkable development in the field of genetics. This was due to great technological progress in the laboratory, through mapping and analyzing the human genetic substrate and its association with diseases, and a better understanding of molecular and genetic mechanisms, allowing a more detailed comprehension of the role of genetics and environment in determining the risk of disease. It was inevitable that geneticists would focus their attention on ischemic cardiovascular disease, since this and its underlying pathological processes (atherosclerosis and thrombosis) are the leading cause of morbidity and mortality in the world. ${ }^{1,2}$

\section{Evidence of genetic predisposition to CHD}

CAD comprises a spectrum of clinical diagnoses [e.g..angina, myocardial infarction $(\mathrm{MI})]$ which are caused by atherosclerosis, a pervasive degenerative condition in which lipid and fibrous matrix is deposited in arterial vessel walls to form atheromatous plaques. Rupture of unstable plaques in coronary arteries results in the release of thrombogenic material into the lumen of the vessel leading to coronary thrombosis, vessel occlusion, and subsequent infarction of the myocardium, a critical condition with high mortality. ${ }^{3}$ A genetic predisposition to CHD has been documented for many years through numerous studies, since the CHD phenotype often exhibits a remarkably inheritable pattern.

\section{Familial clustering}

Family history is the most direct evidence for the estimation of genetic susceptibility to CHD. The Framingham study reported that a family history of CHD or stroke is associated with CHD independently of other cardiovascular risk factors, showing a 2.4-fold increase in risk for men and a

Address of Correspondence: Dr. Md. Toufiqur Rahman, Department of Cardiology, National institute of Cardiovascular Diseases, Dhaka, Bangladesh. email: drtoufiq1971@gmail.com 
2.2 -fold increase in risk for women. ${ }^{3}$ Other studies reported that CHD in first-degree relatives raised the risk by 2-3 times more. ${ }^{4,5}$ The extent to which the familial clustering of CAD can be explained by heritable quantitative variation in the classical risk factors such as cholesterol and $\mathrm{BP}$ is contentious, and the small but significant residual familial relative risk after regression on these conventional risk factors suggests the presence of unmeasured genetic risk factors, raising the possibility of a high level of genetic control by a small number of genes that control fundamental physiological systems $6,7,9,11$

\section{Heritability}

A study in Danish twins revealed a higher incidence of CHD and deaths among monozygotic (44\%) rather than dizygotic (14\%) twins. ${ }^{6}$ Another study in twins highlighted the strong genetic basis of CHD and its main complication, myocardial infarction, in addition to other modifiable risk factors. ${ }^{7} \mathrm{Al}$ so, in a study of 20,966 Swedish twins over 36 years, the heritability of fatal incidents was estimated; the risk amounted to 0.57 for men and 0.38 for women. ${ }^{8}$ In the INTERHEART study, a family history of CHD raised the risk to 1.55 after adjustment for age, sex, smoking, and geographic region, while after correction for nine known factors it was slightly reduced to $1.45 .{ }^{9}$ Finally, the PROCAM study also found family history of myocardial infarction to be an independent risk factor. After adjustment for age, systolic blood pressure, cholesterol, smoking, triglycerides, and lipoprotein (a), the risk was $1.67 .{ }^{10}$

Such high heritability are supported by adoption studies showing a relative risk of death in adoptees of 4.5 for death from vascular disease before the age of $50 \mathrm{yr}$ of a biological parent compared to a relative risk of 3.0 for death of an adoptive parent. 12,13

Further evidence for a moderate genetic influence on the risk of dying prematurely in adulthood, with only a small effect of the family environment was shown by higher mortality among biological parents who had children dying in the age range 25 through 64 yr for death from natural causes, infectious causes, vascular causes, and from all causes combined, with no significant effects for the adoptive parents. ${ }^{14}$

Mendelian forms of CAD studies of rare Mendelian forms of CAD have shown how mutations in genes [low density lipoprotein receptor (LDLR), apolipoprotein B (ApoB), proprotein convertase subtilisin/kexin type 9 (PCSK9), low density lipoprotein receptor adaptor protein 1 (ARH), ATPbinding cassette, sub-family G (WHITE), member 5 (ABCG5), cytochrome P450, family, ${ }^{7}$ subfamily A, polypeptide 1 (CYP7A1)] ${ }^{10}$ involved in lowdensity lipoprotein and high-density lipoprotein metabolism/homeostasis can cause premature $\mathrm{CAD}$, but these mutations are thought to explain a relatively minor fraction of familial CAD. However studies of monogenic diseases have helped to unravel the molecular pathways that regulate cholesterol metabolism, resulting in the development of HMG-CoA-reductase inhibitors. ${ }^{15,16}$ Also, studies of two rare genetic lipid disorders, Tangier disease and sitosterolemia, have revolutionized our understanding of sterol transport. 17,18

All these point to a multifactorial polygenic causation of $\mathrm{CAD}$, with evidence that susceptibility genes for CAD might exist that are independent of hypercholesterolaemia or arterial hypertension or diabetes and may act by affecting an intermediate factor such as vascular inflammation, oxidative stress, thrombogenicity, arterial calcification, etc. It is likely that susceptibility genes might be involved in novel biological pathways or be novel genes in well known pathways such as cholesterol metabolism. The search and identification of genetic factors contributing to $\mathrm{CAD}$ is important to increase our understanding of CAD pathophysiology and develop new strategies for risk prediction and intervention.

\section{Genetic architecture of CAD}

The success of CAD gene mapping is dependent on its genetic architecture which refers to the number of disease genes that exist, their allele frequencies, the risks that they confer, and the interactions between multiple genetic and environmental factors. ${ }^{19,20}$

Although the total genetic contribution to $\mathrm{CAD}$ risk can be quantified, the determination of the size and number of contributing effects is impossible without identifying all CAD susceptibility genes. The multiple risk factors for CAD themselves have their own genetic architecture. The heritability of some of the risk factors for CAD are considerable 
- total cholesterol (40 to 60\%), HDL-cholesterol (45 to $75 \%$ ), total triglycerides (40 to $80 \%$ ), body mass index (25 to $60 \%$ ), systolic blood pressure (50 to 70\%), Lp(a) levels (90\%), homocysteine levels (45\%), type 2 diabetes (40 to 80\%), fibrinogen (20 to $50 \%)^{10}$ Also, as CAD is rare before the age of $50 \mathrm{yr}$, it is unlikely to have an effect on reproductive success and hence less likely to have been subject to direct evolutionary selection pressure. Variants that confer susceptibility or protection for CAD might therefore have evolved neutrally in the past, and so could present at a wide range of frequencies.

This is the basis of the Common Disease/Common Variant (CDCV) hypothesis which holds that the genetic variants underlying complex traits occur with a relatively high frequency $(>1 \%)$, have undergone little or no selection in earlier populations and are likely to date back to $>100,000$ years ago. ${ }^{21}$ The other competing model is the Common Disease Rare Variant hypothesis, with an inverse relationship between the magnitude of genetic effect and allele frequency. ${ }^{22}$

This model argues that diseases are common because of highly prevalent environmental influences, not because of common disease alleles in the population. ${ }^{19} \mathrm{~A}$ review of candidate gene associations and recent genome wide association study results support the importance of common alleles in CAD. ${ }^{23,24}$ At odds with this, rare allelic variants of three candidate genes (ABCA1, APOA1, LCAT) that influence HDL levels, were jointly found to make a substantial contribution to the population distribution of HDL levels ${ }^{25,26}$ The most likely scenario would be that the allelic spectrum of the disease variants is the same as the general spectrum of all disease variants. Under this neutral model, although most susceptibility variants are rare with minor allele frequencies (MAF) $<1$ per cent, variants with MAF $>1$ per cent would account for more than 90 per cent of the genetic differences between individuals. It is plausible that these common variants might contribute significantly to those common diseases in which susceptibility alleles might not be under intense negative selection.

\section{Brief history of genetic engineering}

Genetic association studies with cardiovascular disease using DNA markers have been carried out over the last 30 years. The evolution of technology led to a burst of studies, especially during the last five years. In 1970, it was impossible to study human DNA directly, but only via major cytogenetic abnormalities visible microscopically. Nevertheless, many serological markers that were often studied, such as ABO blood groups and types of leukocyte antigens checked for their connection to disease, in fact reflected the level of genetic alterations of DNA and represented it in a way. ${ }^{11}$

The transition from protein representatives tï DNA polymorphisms marked the beginning of the genome study. In 1980, the construction of a genetic linkage map (Southern blot analysis) was attempted using restriction fragment length polymorphisms of DNA (RFLPs). This was printed on radiology film and later converted into gene scores to check for connections with disease. ${ }^{12}$ This method assisted in the mapping of rare diseases on a genetic basis. During the 1980s, Southern blot analysis was succeeded by the revolutionary technique of DNA polymerase chain reaction (PCR), which allowed the replication of small targeted DNA sequences billions of times in a test tube. The arrival of PCR facilitated the analysis of polymorphisms and mutations, reduced the time and cost of genetic studies, and still remain a fundamental laboratory technique. ${ }^{13}$ In 2000 , technological progress provided scientists with the method of the DNA microarray, also called the "single nucleotide polymorphism (SNP) chip", which allowed the simultaneous examination of millions of polymorphisms throughout the genome. Multinational studies, such as the International Hap Map Project, recorded over two million polymorphisms in multinational populations. 14 In 2005, the first computerized DNA microarray appeared, and the technology has been constantly developing ever since. This, combined with high penetration platforms, reached the goal of analyzing the DNA samples of hundreds of thousands of patients, as well as healthy individuals. ${ }^{15}$

\section{Coronary heart disease genetics}

In the last 20 years, with developing improvements in genotyping, several methods have been used to link the influence of a genetic polymorphism with a phenotypic effect. Considering the genetic basis of CHD, it is necessary to distinguish between the rare single gene causes and the common type, also 
called multigenic, whose pathogenesis involves various factors. Multigenic CHD is of significant interest, given the important role it plays in public health. Nevertheless, several elements in our understanding of the common types of genetic variation were derived from research into singlegene diseases characterized by a low incidence of genetic changes and considerable phenotypic effect. This last category includes examples such as hypertrophic or dilated cardiomyopathy, channelopathies (Brugada syndrome and long-QT syndrome), as well as familial dyslipidemias. ${ }^{16}$ Indeed, research into single-gene CHD preceded interest in the multigene type by some decades. With the passage of time, two main methods developed and are listed below.

\section{Candidate gene method}

The candidate gene method was the first and simplest used. It was based on the a priori assumption that a gene is involved in the mechanisms and plays a role in determining an intermediate phenotype, such as in molecular and cellular function through proteins, or a clinically evident result. In this gene, genetic changes occur that affect the abovementioned operations and eventually result in a high risk of CHD. Early studies focused on single polymorphisms and single genes for research and identification of functional changes in the associated proteins. Frequencies of a small number of selected genetic variants were compared between groups of patients (cases) and healthy subjects (controls). ${ }^{17}$ The first candidate gene study in cardiovascular disease was published in 1992. It made clear that homozygotes who carry a deletion in the gene region for angiotensinconverting enzyme, which had already been linked to higher levels of circulating enzyme, are at higher risk of myocardial infarction. This association was much more evident in people who had no other documented (non-genetic) risk factors and supported the significance of studying genetic factors. 18 Subsequently, studies were conducted that included genotyping research on more than a single polymorphism in the same gene. The first such study published addressed two polymorphisms in distant regions of the gene encoding the coagulation factor VII. The results demonstrated that individuals carrying these polymorphisms are at higher risk of myocardial infarction, perhaps through thrombotic activity of the elevated levels of factor VII and its antigens that are present. ${ }^{19}$ In this case, as previously, there are some intermediate endpoints that act as a link between the genotype and the disease. A brilliant example of a single gene disease predisposing to early coronary disease is familial hypercholesterolemia. It is characterized by an excessive plasma concentration of low-density lipoprotein (LDL) cholesterol and early-onset myocardial infarction. It is caused by mutations in genes encoding proteins that regulate LDL plasma levels. ${ }^{20}$ People suffering from familial hypercholesterolemia exhibit eightfold higher mortality compared to the general population between the ages of 20-59 years. ${ }^{21}$ This is mainly due to CHD, and the risk can be reduced to the general population levels by early intensive statin therapy. ${ }^{22}$ Not only patients, but also their relatives should be monitored. If they also carry relevant mutations, various recommendations should be made, including lifestyle changes and an early initiation of statin treatment.

The candidate gene method has several limitations. One of them was monitoring one or a few polymorphisms in a given gene, while many others, seated even thousands of base pairs away from the gene, could also potentially affect the observed effect. Therefore, given the enormous variability of the human genome, genetic variables acting strongly in the determination of the structure and function of proteins could escape linkage to the disease. Moreover, the limited numbers of people monitored in these studies, as well as the heterogeneity of the samples, exhibited a number of false positives, leading to reduced statistical power and questionable publications.

Since 1980, thousands of studies using the candidate gene method have been published without consistent findings. ${ }^{23}$ Many of the early correlations were neither repeated nor confirmed by other researchers. However, genetic variations of a limited number of genes, acting mainly on LDL cholesterol, were repeatedly and reliably associated with the risk of CHD. ${ }^{24}$ In any case, considering an overall review of the results, the limited success of the candidate gene method was not surprising, though it triggered further research on DNA. 


\section{Genome-wide association study}

A genome-wide association study (GWAS) detects any genetic variants throughout the whole human genome, trying to identify genetic correlations with observed traits or diseases. The method does not require a preselected gene, but investigates a huge number of polymorphisms for any possible association between some of them and various phenotypes. A genotyping record is achieved for hundreds of thousands of people in a fast and efficient process. Lately, it is widely used in studies on variations or genes of many complex diseases, including CHD. GWAS uses methods of standard design. People who carry the disease are compared to non-carriers (case-control studies). The frequency or the intensity of appearance of a genetic polymorphism (biallelic gene) is calculated for both carriers and controls. A statistically higher incidence among people who carry the disease marks it as a risk allele. The estimation of this risk is based on calculation of the odds ratio. ${ }^{25}$ The second type of GWAS design includes a quantitative correlation of a trait, such as cholesterol or blood pressure, which is constantly monitored in a sample of the general population. All individuals are divided into genetic categories according to their allele (homozygotes or heterozygotes). The mean value of the quantitative characteristic is statistically compared for each and every genetically defined group (linearly or with correlation coefficient b). The specific polymorphism is the independent variable, while the dependent variable is the trait. The correlation between a genotypic polymorphism and the measurement of the quantitative trait provides an assessment of the effect magnitude. ${ }^{11}$ When specifying the association of a polymorphism with a disease or a disease trait, the position of this variable within the genome is studied as well as the proximal genomic environment, which is described as the place (locus).

The first GWAS studies in CHD were independently and simultaneously published in 2007. All these studies determined only a few polymorphisms linked to CHD, all located in the 9p21.3 genome region. ${ }^{26-28}$ In a short time several groups worldwide confirmed the results regarding the $9 \mathrm{p} 21$ region, while 11 new high-risk genetic variants for CHD were also mapped. These studies made clear that each of these genetic variables could lead to a moderate or minimal risk effect. Besides, the sample size to reliably detect most of these variables should have exceeded that initially planned, according to statisticians. ${ }^{29}$ Responding to this observation, researchers with previously published successful results combined their sources and data into international formations. This partnership was the greatest collaboration in cardiology, since CARDIoGRAM30 focused on a sample of 86,995 people $(22,233$ cases and 64,762 controls) and a validation sample of 56,682 people, while the IBC50K CAD Consortium31 included 57,594 subjects (15,596 cases and 34,992 controls) and a validation sample of 57,594 people. Thereby, new genetic risk variables were detected and others were confirmed, while in a short period of 5 years a total of 36 genetic risk variables associated with a high risk for CHD were identified. Seven of them act through lipoproteins, two through blood pressure, and one on myocardial infarction, while 23 genetic loci act independently of all known risk factors. 32 These variables are more common than expected, since more than half appear in $>50 \%$ of the population and ten of them appear in $>75 \%$ of the population. The majority of genetic variables exhibit minimal risk, ranging from $6 \%$ to $17 \%$, whereas $>66 \%$ of them express their risk independently of other known risk factors, such as cholesterol. The fact that 23 of the 36 risk variables for CHD do not act through any of the known risk factors indicates unknown mechanisms that need to be elucidated. ${ }^{33}$

In another review of 35 common variables that affect CHD, the risk for disease did not exceed 13\% of the total heritability of CHD. ${ }^{34}$ All these findings suggest that there are still many things to be discovered. However, preventive treatment of CHD is unlikely to be achieved without complete clarification, consideration and prevention of the genetic predisposing factors. The advantage of DNA risk variables versus blood biomarkers is that they are not altered during an individual's lifetime; they are not affected by age, or by meals, medications or environmental stimuli. Therefore, the DNA genotype at birth is stable and can be used for the entire lifetime. All GWAS studies for CHD used the phenotype of either e" $50 \%$ damage to one or more coronary vessels, identified by angiography, or proven myocardial infarction. However, some genes predispose people particularly to the process 
of atherosclerosis and others to myocardial infarction. Regarding the genetic variables, the extensively studied 9p21 acts on the vascular wall and contributes to the pathogenesis of atherosclerosis, without being involved in the acceleration and occurrence of myocardial infarction. ${ }^{34-36}$ Only 2 of 11 polymorphisms associated with myocardial infarction affect wellknown traditional risk factors and involve LDL cholesterol and lipoprotein(a).37 A recent study proved that a risk variable for myocardial infarction is associated with the DNA position responsible for ABO blood group. GWAS studies consistently confirm that genes defining blood groups A and B are associated with myocardial infarction, having no connection with coronary atherosclerosis. ${ }^{38}$ This raises a concern as to whether, in the future, individuals with blood groups $\mathrm{A}, \mathrm{B}$, or $\mathrm{AB}$ should receive antiplatelet therapy preventively, especially those who suffer from CHD or have undergone cardiac surgery. It is clear that clinical studies are required to assess the scale of the problem and the possibility of reversing the effects through the application of this particular treatment.

Clinical Implications for Genetic Testing and Management of Coronary Artery Disease

There are multiple risk variants occurring in the majority of population that act independently of known risk factors. Second, comprehensive prevention of CAD is unlikely without modifying genetic predisposition. It will require more information and time to explore these genetic risk variants, and perhaps function will have to be determined before implementation. However, 9p21 compares favorably with conventional risk factors. The 9p21 variant in individuals with early onset of CAD has a 2-fold increase risk in homozygotes and a $50 \%$ increase risk in heterozygotes. ${ }^{26,27}$ This risk is equivalent to the 2 -fold increased risk of smoking, ${ }^{39}$ the $30 \%$ increased risk of a SD increase in low-density lipoprotein cholesterol (LDL-C) or decrease in high-density lipoprotein cholesterol, ${ }^{40}$ or the $40 \%$ increased risk associated with a $10-\mathrm{mm}$ increase in blood pressure. ${ }^{41}$

The technical barrier to testing for hundreds of genetic risk factors has promptly been overcome using either blood or saliva as the source of DNA. One approach that could be considered currently is using the independent risk factor of 9p21 for more aggressive treatment in lowering LDL-C. Currently, according to the guidelines, LDL-C should be decreased to $160 \mathrm{mg} / \mathrm{dL}$ if 1 risk factor is present, to $140 \mathrm{mg} / \mathrm{dL}$ if 2 risk factors are present, and to $<100 \mathrm{mg} / \mathrm{dL}$ if more risk factors are present.

The recommendation in an individual with elevated LDL-C, with no other conventional risk factors but havinge" 1 genetic risk factors such as 9p21, could be to categorize the individual into a higher risk category, resulting in more intensive treatment of LDL-C.

\section{Future perspectives}

GWAS studies had considerable interest in identifying new genetic loci for CHD and myocardial infarction, whereas the responsible genes provided new insights into the genetic architecture of these diseases. Although these studies highlighted powerful markers and offered immediate improvement in the determination of risk for patients with $\mathrm{CHD}$, the original expectations have now come to a halt. Regarding the findings so far, there are limitations based on the small percentage of disease risk, the unknown mechanisms of most variables, the possible interactions among them, and the discovery of new ones. These clearly give the impression that there are many missing parts to complete the puzzle of heredity. After more than 5 years of high-profile cellular and molecular experiments, the mechanism through which the 9p21.3 locus increases the risk of CHD remains undetermined..$^{42,43}$ Further clarification and crucial information is expected as a result of nextgeneration DNA sequencing techniques. ${ }^{44-49}$ In this kind of genome sequencing, platforms from either the whole genome or selected regions of it are cut into small fragments, thus providing the potential for several different readings. This new strategy calls for the development of new bioinformatics tools to enable analyses of the large amount of resulting data. Now a day's various bioinformatics programs for different sequencing platforms are available. Generally the assessment of genetics in CHD is still a tough, yet highly promising conundrum. Constantly increasing value through research and development will ultimately allow routine genetic screening, aiming at an attempt to reduce CHD risk using pharmaceutical or other approaches. 


\section{Conclusion}

GWAS have confirmed that genetics plays a role in predisposition toward CAD. The genetic risk variants are very common, with some occurring in $>75 \%$ of the population. The major finding that is likely to have the greatest impact on treatment of $\mathrm{CAD}$ is the observation that 23 of the 36 genetic risk variants act through as yet unknown mechanisms. This has the potential to provide new targets for drug development. It will also elucidate new knowledge of the biology of atherosclerosis and MI. The 23 variants acting through unknown mechanisms may act through only 1 or 2 pathways. A major pathway such as the cholesterol pathway is likely waiting to be discovered. The technology barrier for rapid $(<60$ min) genotyping of hundreds of genes simultaneously on a single blood sample has already been eliminated. The application of routine genetic testing will occur gradually, and perhaps appropriately so, as laws will have to evolve to protect privacy and it will require some time to educate physicians and society of the implications of genetics in medical management.

\section{Conflict of Interest - None.}

\section{References}

1. Lopez A, Mathers C, Ezzati M, et al. Global Burden of Disease and Risk Factors. World Health Organisation, 2008.

2. Go AS, Mozaffarian D, Roger VL, et al; American Heart Association Statistics Committee and Stroke Statistics Subcommittee. Heart disease and stroke statistics2013 update; a report from the American Heart Association. Circulation 2013; 127: e6-e245.

3. Watkins H, Farrall M. Genetic susceptibility to coronary artery disease: from promise to progress. Nat Rev Genet 2006; 7: 163-173.

4. Hopkins PN, Williams RR, Kuida H, et al. Family history as an independent risk factor for incident coronary artery disease in a high-risk cohort in Utah. Am J Cardiol 1988; 62: 703-707.

5. Colditz GA, Rimm EB, Giovannucci E, Stampfer MJ, Rosner B, Willett WC. A prospective study of parental history of myocardial infarction and coronary artery disease in men. Am J Cardiol 1991; 67: 933-938.

6. Lloyd-Jones DM, Nam BH, D’Agostino RB, Sr., Levy D, Murabito JM, Wang TJ, et al. Parental cardiovascular disease as a risk factor for cardiovascular disease in middle-aged adults: a prospective study of parents and offspring. JAMA 2004; 291: 2204-2211.
7. Myers RH, Kiely DK, Cupples LA, Kannel WB. Parental history is an independent risk factor for coronary artery disease: the Framingham Study. Am Heart J 1990; 120: 963-969.

8. Zdravkovic S, Wienke A, Pedersen NL, Marenberg ME, Yashin AI, De Faire U. Heritability of death from coronary heart disease: a 36-year follow-up of 20966 Swedish twins. J Intern Med 2002; 252: 247-254.

9. Yusuf S, Hawken S, Ounpuu S, et al. INTERHEART Study Investigators. Effect of potentially modifiable risk factors associated with myocardial infarction in 52 countries (the INTERHEART Study): case-control study. Lancet 2004; 364: 937-952.

10. Cooper JA, Miller GJ, Humphries SE. A comparison of the PROCAM and Framingham point-scoring systems for estimation of individual risk of coronary heart disease in the Second Northwick Park Heart Study. Atherosclerosis 2005; 181: 93-100.

11. Allayee H, Ghazalpour A, Lusis AJ. Using mice to dissect genetic factors in atherosclerosis. Arterioscler Thromb Vasc Biol 2003; 23 : 1501-1509.

12. Botstein D, White RL, Skolnick M, Davis RW. Construction of a genetic linkage map in man using restriction fragment length polymorphisms. Am J Hum Genet 1980; 32: 314-331.

13. Sorensen TI, Nielsen GG, Andersen PK, Teasdale TW. Genetic and environmental influences on premature death in adult adoptees. $N$ Engl J Med 1988; 318: 727 732 .

14. Petersen L, Nielsen GG, Andersen PK, Sorensen TI. Case-control study of genetic and environmental influences on premature death of adult adoptees. Genet Epidemiol 2002; 23: 123-132.

15. Roberts R, Stewart AF, Wells GA, Williams KA, Kavaslar $\mathrm{N}$, McPherson R. Identifying genes for coronary artery disease: An idea whose time has come. Can J Cardiol 2007; 23 Suppl A: 7A-15A.

16. Damani SB, Topol EJ. Future use of genomics in coronary artery disease. J Am Coll Cardiol 2007; 50: 1933-1940.

17. Pullinger CR, Hennessy LK, Chatterton JE, et al. Familial ligand- defective apolipoprotein B. Identification of a new mutation that decreases LDL receptor binding affinity. J Clin Invest 1995; 95: 1225-1234.

18. Cambien F, Poirier O, Lecerf L, et al. Deletion polymorphism in the gene for angiotensin-converting enzyme is a potent risk factor for myocardial infarction. Nature 1992; 359: 641-644.

19. Iacoviello L, Di Castelnuovo A, De Knijff P, et al. Polymorphisms in the coagulation factor VII gene and the risk of myocardial infarction. N Engl J Med 1998; 338: 79-85.

20. Swerdlow DI, Holmes MV, Harrison S, Humphries S. The genetics of coronary heart disease. $\mathrm{Br} \mathrm{Med} \mathrm{Bull}$ 2012; 102: 59-77. 
21. Lander ES. The new genomics: global views of biology. Science 1996; 274: 536-539.

22. Neil A, Cooper J, Betteridge J, et al. Reductions in allcause, cancer, and coronary mortality in statin-treated patients with heterozygous familial hypercholesterolaemia: a prospective registry study. Eur Heart J 2008; 29: 2625-2633.

23. Lohmueller KE, Pearce CL, Pike M, Lander ES, Hirschhorn JN. Meta-analysis of genetic association studies supports a contribution of common variants to susceptibility to common disease. Nat Genet 2003; 33: 177-182.

24. Mayer B, Erdmann J, Schunkert H. Genetics and heritability of coronary artery disease and myocardial infarction. Clin Res Cardiol 2007; 96: 1-7.

25. Schunkert H, Erdmann J, Samani NJ. Genetics of myocardial infarction: a progress report. Eur Heart $J$ 2010; 31: 918-925.

26. McPherson R, Pertsemlidis A, Kavaslar N, et al. A common allele on chromosome 9 associated with coronary heart disease. Science 2007; 316: 1488-1491.

27. Helgadottir A, Thorleifsson G, Manolescu A, et al. A common variant on chromosome 9p21 affects the risk of myocardial infarction. Science 2007; 316: 1491-1493.

28. Samani NJ, Erdmann J, Hall AS, et al. Genomewide association analysis of coronary artery disease. $N \mathrm{Engl}$ J Med 2007; 357: 443-453.

29. Roberts R, Stewart AF. Genes and coronary artery disease: where are we? J Am Coll Cardiol 2012; 60: 1715-1721.

30. Large-scale gene-centric analysis identifies novel variants for coronary artery disease. PLoS Genet 2011; 7: e100-e260.

31. Roberts R, Stewart AF. Genetics of coronary artery disease in the 21st century. Clin Cardiol 2012; 35: 536540 .

32. Peden JF, Farrall M. Thirty-five common variants for coronary artery disease: the fruits of much collaborative labour. Hum Mol Genet 2011; 20: R198-205.

33. Dandona S, Stewart AF, Chen L, et al. Gene dosage of the common variant 9p21 predicts severity of coronary artery disease. J Am Coll Cardiol 2010; 56: 479-486.

34. Patel RS, Su S, Neeland IJ, et al. The chromosome 9p21 risk locus is associated with angiographic severity and progression of coronary artery disease. Eur Heart J. 2010; 31: 3017-3023.

35. Ardissino D, Berzuini C, Merlini PA, et al. Influence of 9p21.3 genetic variants on clinical and angiographic outcomes in early-onset myocardial infarction. $J \mathrm{Am}$ Coll Cardiol 2011; 58: 426-434.

36. Reilly MP, Li M, He J, et al. Identification of ADAMTS7 as a novel locus for coronary atherosclerosis and association of $\mathrm{ABO}$ with myocardial infarction in the presence of coronary atherosclerosis: two genome-wide association studies. Lancet 2011; 377: 383-392.
37. Paynter NP, Chasman DI, Buring JE, Shiffman D, Cook NR, Ridker PM. Cardiovascular disease risk prediction with and without knowledge of genetic variation at chromosome 9p21.3. Ann Intern Med 2009; 150: 65-72.

38. Muehlschlegel JD, Liu KY, Perry TE, et al. Chromosome 9p21 variant predicts mortality after coronary artery bypass graft surgery. Circulation 2010; 122: S60-S65.

39. Schaefer EJ, Lamon-Fava S, Jenner JL, et al. Lipoprotein(a) levels and risk of coronary heart disease in men. The Lipid Research Clinics Coronary Primary Prevention Trial. JAMA 1994;271:999 - 1003.

40. Di Angelantonio E, Sarwar N, Perry P, et al. the Emerging Risk Factors Collaboration. Major lipids, apolipoproteins, and risk of vascular disease. JAMA 2009;302:1993 - 2000 .

41. Sesso HD, Stampfer MJ, Rosner B, et al. Systolic and diastolic blood pressure, pulse pressure, and mean arterial pressure as predictors of cardiovascular disease risk in men. Hypertension 2000;36:801 - 807.

42. Paynter NP, Chasman DI, Paré G, et al. Association between a literature-based genetic risk score and cardiovascular events in women. JAMA 2010; 303: 631637.

43. Ripatti S, Tikkanen E, Orho-Melander M, et al. A multilocus genetic risk score for coronary heart disease: case-control and prospective cohort analyses. Lancet 2010; 376: 1393- 1400.

44. Thanassoulis G, Peloso GM, Pencina MJ, et al. A genetic risk score is associated with incident cardiovascular disease and coronary artery calcium: the Framingham Heart Study. Circ Cardiovasc Genet 2012; 5: 113-121.

45. Visel A, Zhu Y, May D, et al. Targeted deletion of the 9p21 non-coding coronary artery disease risk interval in mice. Nature 2010; 464: 409-412.

46. Harismendy O, Notani D, Song X, et al. 9p21 DNA variants associated with coronary artery disease impair interferon-ã signalling response. Nature 2011; 470: 264268.

47. Maouche S, Schunkert H. Strategies beyond genomewide association studies for atherosclerosis. Arterioscler Thromb Vasc Biol 2012; 32: 170-181.

48. Faita F, Vecoli C, Foffa I, Andreassi MG. Next generation sequencing in cardiovascular diseases. World $J$ Cardiol 2012; 4: 288-295.

49. Padmanabhan S, Hastie C, Prabhakaran D, Dominczak A. Genomic approaches to coronary artery disease. Indian J Med Res 2010; 132: 567-578.

50. Bampali K, Mouzarou A, Lamnisou K, Babalis D . Genetics and Coronary Artery Disease: Present and Future . Hellenic J Cardiol 2014; 55: 156-163.

51. Roberts R. Genetics of Coronary Artery Disease. Circ Res 2014;114: 1890-1903.

52. Lieb W, Vasan R. Genetics of Coronary Artery Disease. Circulation 2013;128:1131-1138 